\title{
Simulation of flow and modelling the residence time distribution in a continuous two impinging liquid-liquid streams reactor using the Monte Carlo Technique
}

\author{
M. Sohrabi \& E. Rajaie \\ Amirkabir University of Technology, \\ Department of Chemical Engineering, Iran
}

\begin{abstract}
In the present study, a stochastic model for the residence time distribution (RTD) in a coaxial counter current liquid-liquid impinging streams system has been developed. Simulations of droplets movements in the impinging spray systems determine the residence time distribution. Droplets dynamics has been formulated from the Boltzmann equation using direct simulation Monte Carlo (DSMC) method. The data predicted from the model has been correlated with the experimental results obtained from a coaxial counter current two impinging liquid-liquid steams apparatus. The reactor consisted of a cylindrical vessel made of Pyrex glass, length $60 \mathrm{~cm}$ and internal diameter $14 \mathrm{~cm}$. The apparatus was equipped with two circular plates placed at the two ends of the reactor. Thus the length of the contact compartment could be varied by moving the plates away from or towards each other. Water and toluene were used as two immiscible liquid streams. These were sprayed into the reactor by applying pressurized nitrogen via special ducts, placed behind the feed nozzles. The degree of agreement between the experimental results and those predicted from the model was $85 \%$.
\end{abstract}

Keywords: impinging streams reactor, residence time distribution, Monte Carlo method, Boltzmann relation, Navier-Stokes equation. 


\section{Introduction}

One of the important operations in chemical engineering is the mass transfer between immiscible phases. In an impinging streams apparatus a unique flow behavior is utilized by which the transfer processes in heterogeneous systems is intensified [1]. In such systems two feed streams, flowing parallel or counter currently collides with each other at a zone in which the two streams impinge.

Impinging streams systems, first proposed by Elperin [2], have been applied as some suitable devices for enhancing mass and heat transfer processes in flowing gas-liquid, gas-solid and liquid-liquid emulsions. In such apparatus, two droplet-laden gaseous jets flowing in opposite directions are allowed to impinge. Some of the multiphase reactions carried out in two impinging streams reactors include two-phase mono-nitration of toluene [3], chemical absorption of $\mathrm{CO}_{2}$ gas in both sodium hydroxide [4] and mono ethanolamine solutions [1] and isomerization of glucose to fructose by an immobilized enzyme [5]. Impinging streams systems have been successfully applied to other chemical processes such as dissolution and mixing as well as mass and heat transfer operations [6-9]. In the present study a stochastic model for the residence time distribution (RTD) of the aqueous phase within a coaxial counter current two liquid-liquid impinging streams contactor (TISC) has been carried out. In addition a comparison has been made between the experimentally determined RTD data and those predicted from the model.

\section{Contactor system}

The experimental apparatus used in the present study is shown in Fig. 1. The contactor consists of a cylindrical vessel made of Pyrex glass, length $60 \mathrm{~cm}$ and internal diameter $14 \mathrm{~cm}$. The contactor is equipped with two circular plates made of "Teflon" placed at the two ends of the contactor. Thus the length of the contact compartment can be varied by moving the plates away from or towards each other.

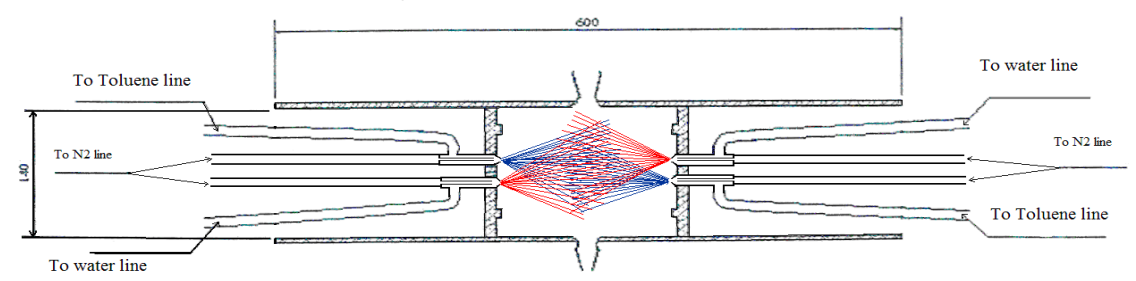

Figure 1: $\quad$ Contactor dimensions (figures are in $\mathrm{mm}$ ).

The two immiscible liquids (toluene and water) were kept in separate glass containers and fed to the contactor via four identical feed nozzles made of glass, situated at the two ends of the vessel. Liquids were transported to the nozzles 
using metering pumps. The liquid streams were sprayed into the contactor by applying pressurized nitrogen via special ducts, placed behind the feed nozzles. In figure 2 , the diagram of a feed nozzle is shown. As it may be observed from this figure, there is no mixing of fluids within the nozzle. The mixing is occurred only at the exit of the system. In each end of the contactor two identical feed nozzles were installed spraying two different liquids towards the impingement zone.

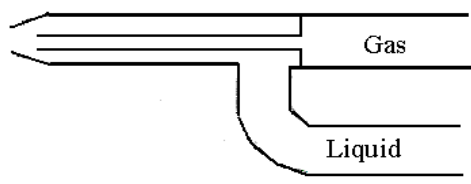

Figure 2: $\quad$ Diagram of a feed nozzle.

Operating conditions are shown in Table 1.

Table 1: Operating conditions of the impinging streams contactor.

\begin{tabular}{|c|c|c|c|c|}
\hline $\begin{array}{c}\text { Temperature } \\
\left({ }^{\circ} \mathrm{C}\right)\end{array}$ & $\begin{array}{c}\text { Distance } \\
\text { between the } \\
\text { two feed } \\
\text { nozzles } \\
(\mathrm{cm})\end{array}$ & $\begin{array}{c}\text { Flow rate of } \\
\text { nitrogen } \\
\left(\mathrm{cm}^{3} / \mathrm{min}\right)\end{array}$ & $\begin{array}{c}\text { Toluene flow } \\
\text { rate } \\
\left(\mathrm{cm}^{3} / \mathrm{min}\right)\end{array}$ & $\begin{array}{c}\text { Water flow } \\
\text { rate } \\
\left(\mathrm{cm}^{3} / \mathrm{min}\right)\end{array}$ \\
\hline 20.1 & 25 & 1300 & 320 & 325 \\
\hline
\end{tabular}

In order to determine the suitable positions and angles for the feed nozzles at which stable jets of liquids would be established, a number of experiments were performed.

This experimental set up allows consideration of the effects of changing certain pertinent parameters of the system on the residence time distribution of materials and hence on the extent of the heterogeneous liquid-liquid mixing and reaction.

\section{Results and discussion}

\subsection{Measurement of the residence time distribution of aqueous phase}

To determine the residence time distribution of the aqueous phase within the contactor, the following experiment was performed. Toluene and distilled water were fed to the contactor via spray nozzles. At a time, a change from the water stream to one of water containing a mineral salt (potassium dichromate) with known concentration and colour intensity $\left(\mathrm{C}_{\mathrm{o}}\right)$ was rapidly performed (step input). Samples at the outlet of the contactor were collected, using a circular vessel divided into 24 segments with equal volumes, placed under the exit port 
and rotated at a pre-set speed applying an electric motor. Successive samples were obtained at equal time intervals by this method. Change in the rotating speed altered the time intervals between the sample collections. The content of each segment was transferred to a separating funnel. The aqueous phase was separated from the organic layer and the concentration (colour intensity) of the former was measured (C), using a UV spectrophotometer. From a plot of $\mathrm{C} / \mathrm{C}_{\mathrm{o}}$ versus time, the RTD data were determined.

\subsection{Developing a stochastic model for the residence time distribution of the aqueous phase in TISC}

\subsubsection{Gas flow simulation}

Gas flow pattern is required to simulate the droplet movement. By the velocity pattern, the drag force which affects the droplet velocity may be estimated.

Complete solution of Navier-Stokes equation for such a system can provide the necessary data, for this pressure, although the approximate solution of this equation (analytical relations) for free jet may be also used.

In the present study the Navier-stokes equation has been solved, using the SIMPLE method and a mesh system shown in figure 3.

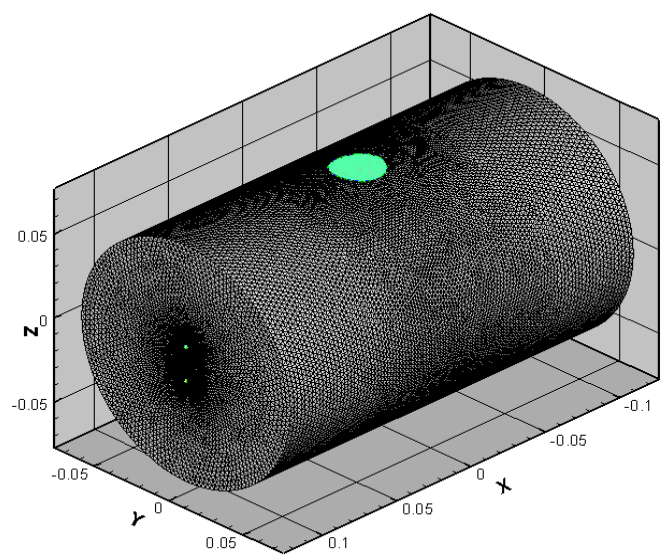

Figure 3: Pyramid mesh for the contactor.

The gas stream is assumed to be an incompressible flow (This assumption is valid for the fluid flows having Mach number lower than 0.3).

Calculation of pressure contours is the main problem in this kind of fluid flow and the SIMPLE method seems to be a way by which the pressure profile under these conditions may be estimated [10]. The results are shown in figure (4). 


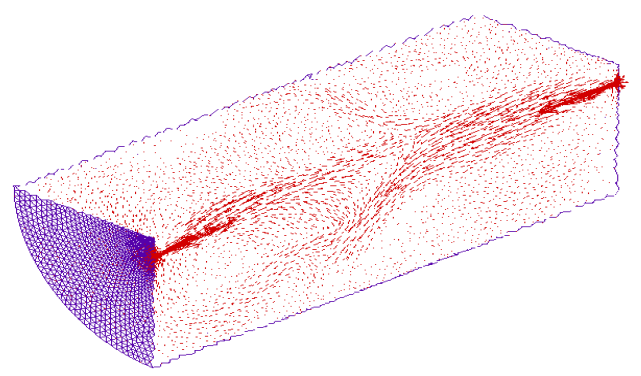

Figure 4: Velocity vectors and streamlines.

\subsubsection{Droplet simulation}

An analogy between the droplet collisions in emulsions and the molecular collisions, described in the kinetic theory of gases, enables the application of the Boltzmann equation to the droplets, as first suggested by Pai [11].

In this work, the direct simulation Monte Carlo (DSMC) method, first proposed by Bird [12] for solving the Boltzmann equation in molecular gas dynamics, is used for modeling the droplet interactions in dense sprays.

\subsection{Mathematical formulations}

The Boltzmann equation may be written as,

$$
\frac{\partial}{\partial t}(n f)+V \cdot \frac{\partial}{\partial \mathrm{r}}(n f)+\mathrm{F} \cdot \frac{\partial}{\partial v}(n f)=\int_{-\infty}^{+\infty} \int_{0}^{4 \pi} n^{2}\left(f^{*} f_{1}^{*}-f f_{1}\right) V_{\mathrm{r}} \sigma d \Omega d V_{1}
$$

In this equation, $\mathrm{n}$ is the number density of droplets, $f$ is the velocity probability distribution function of droplet of class having the velocity $V, f_{1}$ is the velocity distribution function of the droplet of class having the velocity $V_{1}, V_{\mathrm{r}}$ is the velocity of a test droplet in the class of droplets having the velocity $V_{1}, f^{*}$, is the post-collision velocity probability distribution function and $f_{1}^{*}$ is the postcollision velocity probability distribution function of the droplet of class having the velocity $V_{1}, F$, is the external force per unit mass and $\Omega$ is the angle in the spherical coordinates.

The key ideas of the DSMC method are: (a) the uncoupling of droplet motions and collisions during a time step $\Delta t_{m}$ i.e. the use of the operatorsplitting technique (b) the simulation of droplet collisions by disregarding droplet position coordinates within spatial coils: and (c) the simulation of fewer droplets than those present in the real flow, while normalizing the collision cross-section so that the collisions rate is not changed. Assumption (a) is valid when $\Delta t_{m}$ is smaller than the time between collisions, and larger than that of collision duration, and assumption (b) is valid provided that the cell is so small that the spatial variation of flow variables in the cell is negligible. Assumption 
(c) may not be necessary for dilute emulsion flow, due to relatively small droplet number densities; in the present work, however, due to the formation of large number of fragments, the implementation of this procedure is required.

Under the assumptions listed above the DSMC method for the solution of Boltzmann kinetic equation, describing the flow of gas and droplets emulsions can be formulated as follows. The flow system is divided into equal-volume cells. Simulated droplets are distributed in the system, with their positions, sizes and velocities sampled from the initial distribution function. When a stationary kinetic equation is solved, the initial distribution function is chosen arbitrarily. The droplets population is normalized such that each $K_{f}$ droplets of identical size in the real system is substituted by a single droplet in the simulation, having the same diameter as these droplets. The collision cross-section for each such simulated droplet is accordingly increased by the factor $K_{f}$ so as to preserve the true collision rate. Provided that the droplet distribution function at time $(n-1)$ $\Delta t_{m}$ is determined, the distribution function at time $n \Delta t_{m}$ maybe calculated as follows. Droplets are allowed to move in the system, without colliding with each other, for a time interval $\Delta t_{m}$, with each droplet's subsequent position, velocity and diameter are determined from the droplet equation of motion. Within the time interval $\Delta t_{m}$, a droplet may encounter a boundary either an open boundary, through which it leaves the system or a wall, onto which it sticks. Following the collisionless flow, droplets are allowed to collide with each other. The droplet population is discredited by location and size such that the number of droplets of type $k$ in cell $m, N_{m, k}$ having a volume $V_{m}$, is given as,

$$
N_{m, k}(t)=\int_{V_{m}} d r \int_{0}^{\infty} d v \int_{\delta_{k}^{s}}^{\delta_{k}^{l}} d \delta f(r, v, \delta, t)
$$

Where, $\delta_{k}^{s}$ and $\delta_{k}^{l}$ are the lower and upper diameter limits, respectively, for droplets of type $k$. The total number of droplets in cell $\mathrm{m}$ is,

$$
N_{m}=\sum_{k=1}^{s} N_{m, k}
$$

In the stochastic model it has been assumed that collision durations are negligible, so that droplet motion can be described as a free motion, disrupted instantaneously by collisions. For high collision velocity, which is characteristic of impinging streams systems, shorter collision durations may be expected. Another assumption made was that droplets are spherical and the aerodynamic break up due to gas shear during free droplet motion is negligible. Borisov et al. [13] investigated experimentally the break up of single droplets moving in a gas flow. Their results show that droplet break up dose not occur when Weber number $<6$. When only fluid drag acts upon droplets during $\Delta t_{m}$, droplet trajectory is calculated by integrating the following ordinary differential equation,

$$
\frac{d^{2} r}{d t^{2}}=0.75 C_{D}\left[\rho_{g}\left(\rho_{p} \delta\right)\right]|U-v|(U-v)+g
$$


where, $U$, is the gas velocity at position $r$ that is determined according to the procedure presented in gas flow simulation section, $\mathrm{g}$, is the gravity acceleration and $C_{D}$, is the gas drag coefficient for liquid droplet flowing in a gas and may be determined from the following relation proposed by Hestroni [14].

$$
\begin{aligned}
& C_{D}=\left\{\begin{array}{lc}
8(3 \Theta+2) /\left[\operatorname{Re}_{p}(\Theta+1)\right] & \text { for } \operatorname{Re}_{p}<2 \\
\left\{14.9 / \operatorname{Re}_{p}{ }^{0.78}+\Theta\left[(24 / \operatorname{Re})+\left(4 / \operatorname{Re}_{p}{ }^{1 / 5}\right)\right]\right\} /(\Theta+1) & \text { for } 2<\operatorname{Re}_{p}<500
\end{array}\right\} \\
& \operatorname{Re}_{p}=\rho_{g} \delta|U-v| / \mu_{g} \quad \Theta=\mu_{p} / \mu_{g}
\end{aligned}
$$

In this model the droplet were considered to be spherical and collide with each other at different speeds. In simulation of processes, using the DSMC, two grid systems are normally considered. The first grid system is used to calculate the averages of flow properties. This grid system is chosen to be fine enough in order to increase the computational accuracy. The grid system is refined until the variations of the flow properties are not substantial (the variations of the flow properties should be less than 2\%). The second grid system (Fig. 5) is selected to be extremely fine (the mesh size is equal to 0.2 times of the mean free path of the droplets) so that the collision of droplets could be controlled within each mesh with high accuracy. The grid system chosen in this study consisted of 88 divisions in $\mathrm{X}$ direction, 54 divisions in $\mathrm{Y}$ direction, 54 divisions in $\mathrm{Z}$ direction (totally 256608 meshes) and the total number of model droplets was 3,800,000. Each model droplet consists of $K_{f}=70$ real droplets. The size of the mesh is in order of the mean free path of droplets and the time step in the simulation process is chosen to be 0.2 times the collision time [12].

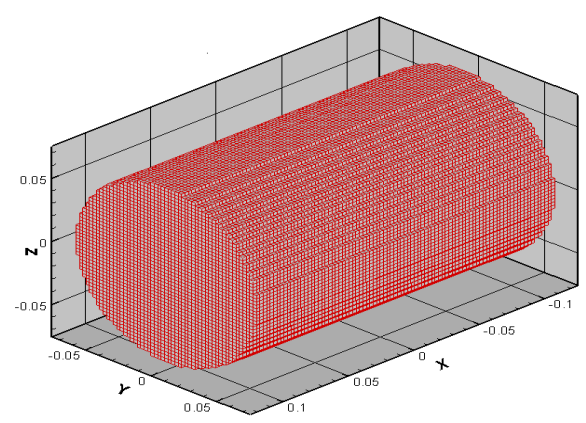

Figure 5: Contactor mesh for DSMC simulation.

The droplets are distributed in the mesh system according to the normal distribution. The initial velocity of the droplets is chosen based upon the velocity of gas jets in the flow field. Then as time passes the new position of the droplets is designated. The collisions of the model droplets are occurred based on the pattern put forward by Bird [12]. Passage of time is continued until the statistical fluctuations of the flow properties attain minimum values.

To implement the DSMC method, spatial cells have to be produced in the first step. The model droplets are placed in the cells with appropriate distribution. 
With regard to local gas velocity, a normal distributed velocity is supposed for droplets. Without any statistical sampling, droplets are moved (NPS times repetition), until the latter attain suitable conditions for statistical sampling. For large domain simulations, this step can be ignored. NPS value definition is dependent on cells conditions; however optimization can not be approached without sufficient experience.

At the next time step, according to each droplet velocity and the extended time step, the droplets are moved and so their new coordinates will be attained. If a droplet passes cell boundary, it will be eliminated from the cell, and a new droplet would be entered to the domain. Furthermore, if in this replacement, the droplet collides with a physical surface, it will be reflected from the surface according to the collision of "surface-droplet model". The index number of cell and sub-cell containing the droplet is defined; and then the probability droplet collision is investigated based on the model and if there is any collision, the velocity and location of the droplet will be modified. After NIS times repetition of movements, indexing, and collision investigations, statistical sampling should be done, the output of the program will be provided after NPT times repetition of sampling. This output is corrected up to NPT times.

The expected number of collisions in a cell, during a time interval $\Delta t_{m}$ is given as,

$$
N_{c}=0.5 n^{2} \overline{\sigma_{T} c_{r}}
$$

The probability $\mathrm{P}$ of collision between two simulated droplets over the time interval $\Delta t_{m}$ is equal to the ratio of the volume swept out by their total crosssection moving at the relative speed between the droplets to the volume of the cell, i.e.,

$$
P=F_{N} \sigma_{T} c_{r} \Delta t / v_{c}
$$

Maximum efficiency is achieved if the fraction is such that the maximum probability becomes unity. The fraction is given by,

$$
P_{\max }=F_{N}\left(\sigma_{T} c_{r}\right)_{\max } \Delta t / v_{c}
$$

In the above equation $\sigma_{T}$ is collision cross section and $v_{c}$ is the relative droplet velocity.

The average number of real droplet in the cell is $n v_{c}$ and the average number of simulated droplets is $N=n v_{c} / F_{N}$, where, $n$, is the number density in liquid phase. full set of collisions could be calculated by selecting in turn, all $N(N-1) / 2$ pairs in the cell and by computing the collision with probability $\mathrm{P}$.

Pairs are selected from the cell at the time step, and the collision is computed using the $\sigma_{T} c_{r} /\left(\sigma_{t} c_{r}\right)_{\max }$ probability relation given by Bird [12].

In the present work two different liquids (water and toluene) have been used. Therefore, the collisions were between similar or unlike droplets so the method has to be slightly modified. A pair of colliding droplets of type $\mathrm{p}$ and $\mathrm{q}$ is sampled from the possible pairs of these types with the above probability. This 
term has been substituted for $\sigma_{T} c_{r} /\left(\sigma_{t} c_{r}\right)_{\max }$ in all related equations. Simulation results for these droplets are shown in figures 6-8.

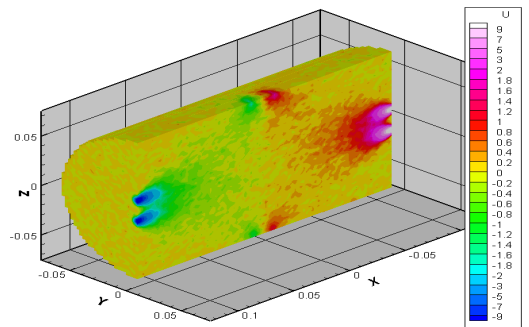

Figure 6: Droplets velocities in $\mathrm{X}$ direction.

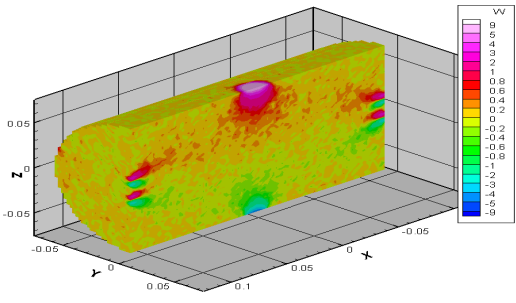

Figure 7: Droplets velocities in $\mathrm{Y}$ direction.

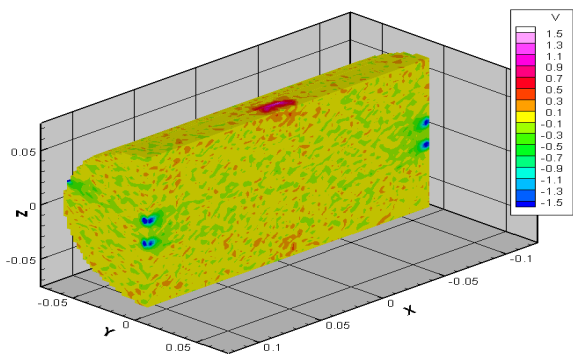

Figure 8: Droplets velocities in $\mathrm{Z}$ direction.

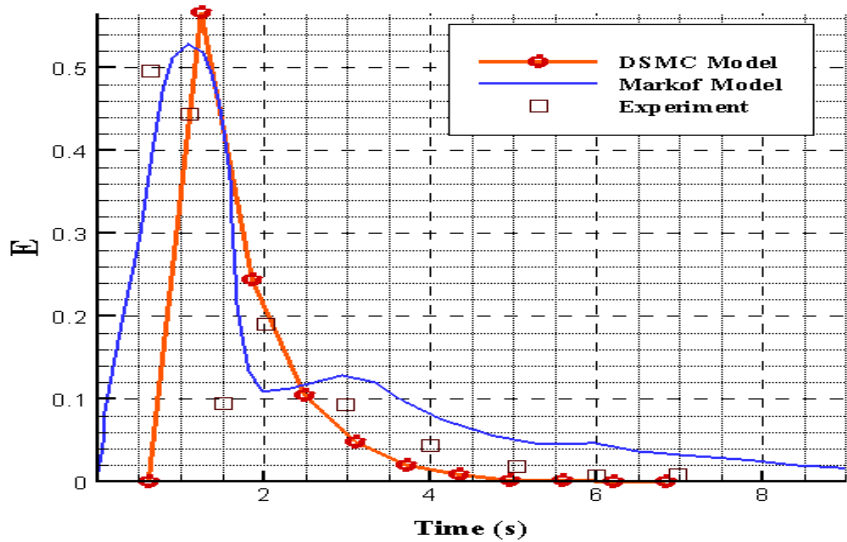

Figure 9: A typical residence time distribution data. 
A typical experimental residence time distribution (RTD) curve obtained for the continuous two impinging streams contactor and those predicted applying the Markov chain model and DSMC technique are shown in figure 9.

\section{Conclusion}

The Direct Simulation Monte Carlo (DSMC) technique was applied to predict the motion of liquid droplets and residence time distribution in a two impinging streams contactor. This method was found to be more accurate and flexible in prediction of RTD data compare to the Markov chain discrete time formulation applied by Sohrabi et al $[3,5,15]$ in some previous studies. The degree of agreement between the data estimated from the present model and those determined experimentally was within $85 \%$. While in case of Markov formulation the degree of agreement was lower than $75 \%$.

\section{References}

[1] Sohrabi, M., Jamshidi, A.M., Studies on the behaviour and application of the continuous two impinging streams reactors in gas-liquid reactions, $J$. Chem. Tech. Biotechnol. 69, pp. 415 420, 1997.

[2] Tamir, A., Impinging Streams Reactors, Fundamentals and Applications, Elsevier B. V., Amsterdam, The Netherlands, 1994.

[3] Sohrabi, M. Kaghazchi, T. \& Yazdani, F., Modelling and application of the continuous impinging streams reactors in liquid - liquid heterogeneous reactions, J. Chem. Tech. Biotechnol., 58, pp. 363 370, 1993.

[4] Tamir, A., Herskovitz, D., Absorption of $\mathrm{CO}_{2}$ in a new two-impingingstreams absorber, Chem. Eng. Sci. 40, pp. 2149 2160, 1985.

[5] Sohrabi, M., Ahmadi Marvast, M., Application of a continuous two impinging streams reactor in solid-liquid enzyme reactions, Ind. Eng. Chem. Res., 39, pp.1903 1910, 2000.

[6] Tamir, A., Kirton, Y., Chem. Eng. Comm., 50, pp. 241 252, 1987.

[7] Tamir, A., Kirton, Y., Drying Technol., 7, pp. 183 191, 1989.

[8] Tamir, A., Luzzatto, K., Mixing of solids in impinging streams reactor, $J$. Powder Bulk Solids Technol., 9(15), pp. 15 17, 1989.

[9] Tamir, A., Chem. Eng. Progress, 85, pp. 53 67, 1989.

[10] Patankar, S.V., Numerical Heat Transfer and Fluid Flow, Elsevier, B.V., The Netherlands, pp. 123 144, 1993.

[11] Pai, S.I., Sci. Tech. Mech. Appl., 19, pp. 605 621, 1974.

[12] Bird, G.A., Molecular Gas Dynamics and the Direct Simulation of Gas Flows, Oxford University Press, UK, pp. 196 211, 1994.

[13] Borisov, A.A., Gelfand, B., Natanzon, M.S. \& Kossov, O., J. Eng. Phys. 40, pp. 44 49, 1981.

[14] Hetsroni, G., Handbook of Multiphase Systems, Hemisphere Inc., Washington, 1982.

[15] Sohrabi, M., Zareikar, B., Modeling of the residence time distribution and application of the continuous two impinging streams reactors in liquidliquid reactions, Chem.Eng.Technol. 28, pp. 61 66, 2005. 